\title{
Sono: Aspectos Profissionais e suas Interfaces na Saúde
}

\author{
Sleep: professionals aspects and health interfaces
}

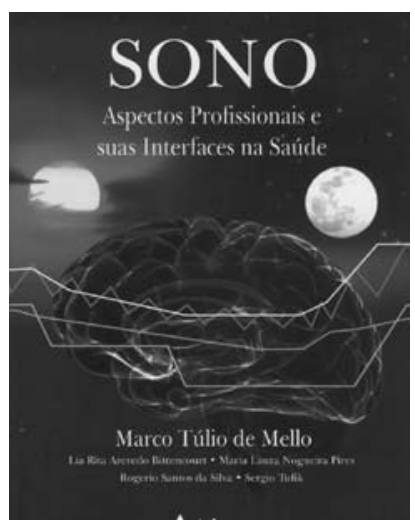

Marco Túlio de Mello, Lia Rita de Azevedo Bittencourt, Maria Laura Nogueira Pires, Rogério Santos da Silva, Sergio Tufik

O livro foi organizado pelo professor Marco Túlio de Mello, orientador de mestrado e doutorado na Universidade Federal de São Paulo (UNIFESP) e Coordenador do Centro Multidisciplinar em Sonolência e Acidentes, contando com os colaboradores Lia Rita de Azevedo Bittencourt, médica, coordenadora clínica do Instituto do Sono, Professora Adjunta de Medicina e Biologia do Sono da UNIFESP; Maria Laura Nogueira Pires pós-doutorada pelo Sleep and Mood Disorders Laboratory da Oregon Health Science University, Estados Unidos, e Pesquisadora do Departamento de Psicologia da UNIFESP; Rogério Santos da Silva, Doutor em Ciências, Biólogo Pesquisador do Departamento de Psicologia da UNIFESP e Sérgio Tufik, Vice-Reitor da UNIFESP, Professor Titular do Departamento de Psicobiologia da UNIFESP, Chefe da Disciplina Medicina e Biologia do Sono da UNIFESP.

Trata-se de uma obra com o título o SONO, com 117 páginas, com boa distribuição entre os nove capítulos, reunindo dez colaboradores. Este conteúdo direciona-se a estudantes, profissionais e pesquisadores da área da saúde e afins. Sua linguagem é clara e objetiva, trazendo inúmeros exemplos, tabelas e figuras que contribuem para as explicações, inclusive para o leitor leigo no assunto.

O capítulo um foi escrito por Claudia Roberta de Castro Moreno (Departamento de Saúde Ambiental da Faculdade de São Paulo), Frida Marina Fischer (Professora Titular do Departamento de Saúde Ambiental, Faculdade de Saúde Pública da Universidade de São Paulo - USP) e Lúcia Retenberg (Pesquisadora Adjunta do Laboratório de Educação em Ambiente e Saúde do Instituto Osvaldo Cruz - FIOCRUZ), as quais abordaram, As jornadas de trabalho dos motoristas profissionais. As autoras exibem as classificações das jornadas dos motoristas (de transportes coletivos e de cargas), que na maioria das vezes têm irregularidades no seu ritmo de sono. Estes motoristas com o objetivo de cumprir o horário combinado com a empresa que contratou o transporte, acabam alterando o seu ciclo vigília-sono, principalmente os de jornadas de trabalho especiais (também denominadas de jornadas irregulares ou outônomas), para que o transporte não seja interrompido até o seu destino. Não raramente estes motoristas são autônomos e não se encaixam nas definições da Organização Internacional do Trabalho (OIT). Ainda neste capítulo, as autoras expõem um estudo sobre as jornadas e o perfil dos motoristas do turno fixo, assim com o perfil dos motoristas de horário irregular e suas evidências das vantagens e desvantagens.

No capítulo dois, elaborado por Maria Laura Nogueira Pires, Camila Fabiana Rossi Squarcini, Marco Túlio de Mello, Lia Rita Azevedo Bittencourt e Rogério Santos da Silva explicam Os ritmos circadianos e os comportamentais: alguns aspectos relevantes no trabaIho por turnos e no noturno. Os autores enfatizam que a intensa luminosidade ao longo do dia (rotação da Terra em torno do seu próprio eixo) é o principal estímulo temporal capaz de sincronizar ritmos circadianos, apesar das intenções sociais, dos horários escolares e do trabalho, da atividade física e do exercício. Os ritmos circadianos da secreção de melatonina (neuro-hormônio produzido pela glândula pineal), da temperatura corporal, do alerta e do tempo de reação, perfazem o ciclo de vinte e quatro horas. Neste estudo, os pesquisadores evidenciaram que os diversos ritmos circadianos acompanham o valor máximo de melatonina coincidindo com o mínimo de temperatura corporal entre $3 \mathrm{~h}$ e $5 \mathrm{~h}$ da manhã, horário em que sentimos mais frio e o sono está na fase mais profunda das vinte quatro horas do dia. 
O capítulo três, escrito por Lia Rita de Azevedo Bittencourt, Rogério Santos da Silva, Maria Laura Nogueira Pires, Marco Túlio de Mello e Sérgio Tufik, O sono e seus distúrbios. Eles referem que os seres humanos gastam um terço de suas vidas dormindo, embora estudos recentes tenham demonstrado que com o passar do tempo, estamos dormindo menos. Dentro do sono, dois estados primários foram definidos com base nos parâmetros fisiológicos: sono sem movimentos rápidos dos olhos (NREM) e com movimentos rápidos dos olhos (REM). Observa-se que o sono NREM esteja provavelmente localizado no hipotálamo anterior, no tálamo, no prosencéfalo basal, no núcleo dorsal da rafe do tronco cerebral, mas os neurônios colinérgicos, responsável por deflagrar e manter o sono REM. Neste mesmo capítulo, os autores inseriram a classificação internacional de distúrbio do sono (CIDS), que possibilita diagnosticar com mais especificidade o tipo de distúrbio, como: as insônias; distúrbios respiratórios relacionados ao sono; hipersonias de origem central não causadas pelos distúrbios do ritmo circadianos do sono; distúrbios respiratórios relacionados ao sono ou outras causas do sono noturno interrompido; distúrbios do ritmo circadiano do sono; distúrbio do movimento relacionado ao sono e outros distúrbios do sono. Nessas classificações, há mais de oitenta tipos de distúrbios do sono para serem diagnosticados.

No capítulo seguinte, Rogério Santos da Silva, Lia Rita de Azevedo Bittencourt, Maria Laura Nogueira Pires, Marco Túlio de Mello e Sérgio Tufik, intitulado À avaliação da sonolência excessiva, dedica-se à anamnese, que é fundamental para identificar o problema do sono; não raramente o parceiro (a) é a peça essencial no auxílio desta investigação. Deve-se dar atenção se há doenças crônicas, como a hipertensão arterial, diabetes mellitus, distúrbios-psiquiátricos ou psicopatológicos, e ou neurológicos, inclusive se o paciente faz uso de drogas/álcool, principalmente durante as duas semanas de avaliação, para um diagnóstico mais eficiente. O que contribui para a avaliação de sonolência excessiva especificamente são as escalas de avaliação, as recomendadas pelos autores são: Escala de Sonolência de Stanford (ESS), Escala de Sonolência de Kalinska (ESK) e Escala de Sonolência de Epworth (ESE). As escalas ESS e ESK são escalas que têm bons resultados no momento da aplicação, porém a ESE pode ser aplicada nas diversas situações. A polissonografia, o teste múltiplo das latências do sono e o teste da manutenção da vigília são de grande utilidade na avaliação da sonolência excessiva objetiva.

No capítulo cinco, Samantha Lemos Paim, Maria Laura Nogueira Pires, Lia Rita de Azevedo Bittencourt, Ruth Ferreira Santos, Rogério Santos da Silva, Sérgio Tufik e Marco Túlio de Mello debatem sobre $\boldsymbol{A}$ sonolência e os acidentes. Enfatizam que a prosperidade de um trabalho, na maioria das vezes, é dependente de algumas mudanças do nosso cotidiano, mesmo que isso implique alterar nossos horários de sono. Em alguns anos, com atividades em horários não convencionais ao ritmo do sono, o trabalhador pode desenvolver distúrbios cardíacos, digestórios que poderão causar a sonolência, o que dificulta a eficiência e a produtividade. Este grupo de pesquisadores demonstrou que a fadiga (cansaço, falta de energia e exaustão) a sonolência (probabilidade aumentada para dormir) e a sonolência excessiva (propensão aumentada para o sono com aumento subjetivo para dormir, tirar cochilos involuntários e ter ataques de sono) são constantes nos trabalhadores por turnos, essencialmente nos do turno noturno (81\%).

O capítulo seis, de autoria de Marco Túlio Mello, Andrea Maculano Esteves, Lia Rita Azevedo Bittencourt, Maria Laura Nogueira Pires, Rogério Santos da Silva e Sergio Tufik, apresenta $\boldsymbol{A}$ sonolência, o rendimento no trabalho e a qualidade de vida. $O$ tema deste capítulo está evidente na sociedade moderna, a qual vive em constante modificação com a presença de dificuldades psicológicas no bem-estar social, aumento nos níveis de estresse e decréscimo no desempenho, além dos distúrbios de saúde, essencialmente do sono, digestivos e mentais, com isso os trabalhadores estão mais propensos à queda da performance física e mental em função da sua atividade profissional. O surgimento dos distúrbios do sono, inclusive a insônia, a sonolência excessiva, apnéia do sono, narcolepsia e síndromes das pernas inquietas, são alguns dos indicativos comuns do desempenho do trabalho por turno e noturno, pois estes alteram a saúde e conseqüentemente a qualidade de vida. 
No capítulo seguinte, escrito por Cibele Crispim, lona Zalcman Znberg, Lia Rita Azevedo Bittencourt, Maria Laura Nogueira Pires, Rogério Santos da Silva, Sérgio Tufik e Marco Túlio de Mello, os autores discutiram A nutrição para os trabalhadores por turno. Os trabalhadores do turno noturno têm mudança nos hormônios que saciam a fome, devido ao desenvolvimento de hábitos alimentares inadequados, elementos que auxiliam na prevalência de algumas patologias como a obesidade, dislipidemias e o diabetes. Os autores deram ênfase também à nutrição e ao ritmo circadiano, a influência endócrina na ingestão alimentar e no sono, a leptina, a insulina e glicose, as recomendações nutricionais para os trabalhadores por turnos, estratégias nutricionais do trabalhador por turno (carboidratos, proteínas e as gorduras, as fibras, sal e o álcool), o fracionamento alimentar, a nutrição e a promoção do sono (cafeína, horário de jantar e aminoácidos)

O capítulo oito elaborado por Franco Noce, Marco Túlio de Melo, Lia Rita Azevedo Bittencourt, Maria Laura Nogueira Pires, Rogério Santos da Silva e Sérgio Tufik, Entendendo o erro humano: uma análise dos processos decisórios nos trabalhadores por turnos. Este tema subdivide-se em: o trabalho por turno; considerações sobre as implicações do trabalho noturno; o sono e o trabalho por turno: considerações gerais e estratégias; as estratégias; os ritmos biológicos; os aspectos cognitivos (percepção, atenção e memória); os fatores que influenciam a memória; a memória humana: retenção e recuperação; a codificação; esquecendo a informação; a resolução dos problemas; os obstáculos; os auxílios à resolução de problemas; os acidentes de trabalho e o erro humano.

No último capítulo é elaborada por Marco Túlio de Melo, Maria Laura Nogueira Pires, Lia Rita Azevedo Bittencourt, Rogério Santos da Silva e Sérgio Tufik $\boldsymbol{A}$ escala de trabalho e a sua relação ou contribuição para o desencadeamento da fadiga e da sonolência excessiva: relato de experiência. Estes autores verificaram que as pessoas que estão envolvidas com o trabalho por turno ou jornadas noturnas, desconhecem o tema. Diversos acidentes ocorridos durante a jornada de trabalho são devidos a fatores relacionados à fadiga e/ ou à sonolência excessiva. Provavelmente, o tipo de acidente mais exposto aos olhos da população seja o acidente de trânsito (automobilístico), pois mundialmente o mesmo é responsável por mais de quinhentas mortes por ano, contra quatrocentos e quarenta mil decorrentes de homicídios e trezentos e vinte mil devido às guerras.

Torna-se evidente em virtude do que os autores expuseram, que este tema deve ser mais esclarecido a comunidade. Nas escolas, nas empresas, através de seminários de saúde pública, socialização em grandes meios de comunicação. O sono é um dos grandes mecanismos de manutenção fisiológica do nosso organismo e auxiliar dos ajustes quando há desequilíbrio orgânico ou psíquico, assim como desenvolvimento de hábitos alimentares inadequados, o que contribui para os distúrbios metabólicos. Também ficou evidente nesta arquitetura do sono a diminuição da atividade profissional, de rendimento nos estudos, no desempenho social, familiar e conjugal. Portanto, é essencial uma ação enérgica das autoridades competentes, para sensibilizar e assegurar a saúde destes trabalhadores, pois uma redução desta vigília contribuiria para uma melhor qualidade de vida

José Carlos Souza

Doutorado em Ciências Médicas pela Universidade Estadual de Campinas (UNICAMP), Professor da Universidade Católica Dom Bosco (UCDB), Campo Grande-MS, Pós-Doutorado pela Universidade de Lisboa, Portugal.

Marcos Antonio Nunes de Araújo

Graduado em Enfermagem pela Universidade Federal de Pelotas-UFPEL, Professor da Universidade Estadual de Mato Grosso do Sul, Mestrando em Psicologia pela Universidade Católica Dom Bosco (UCDB), Especialista em Terapia Intensiva pela Universidade do Contestado, SC (UnC). 